\title{
SLOW STEADY MOTION OF A THERMO-VISCOUS FLUID BETWEEN TWO PARALLEL PLATES WITH CONSTANT PRESSURE AND TEMPERATURE GRADIENTS
}

\author{
J.Srinivas ${ }^{1}$, N.Pothanna ${ }^{2}$, P. Nageswara Rao ${ }^{3}$, N. Ch. Pattabhi Ramacharyulu ${ }^{4}$ \\ ${ }^{1,2,3}$ Department of Humanities \& Science, VNRVJIET, A.P. India \\ ${ }^{4}$ Former faculty, Department of Mathematics, NIT, Warangal, A.P, India. \\ joshi_seenu@yahoo.com,pothareddy81@gmail.com,nageswararao_p@vnrvjiet.in,pattabhi1933@yahoo.com
}

\begin{abstract}
In this paper, the slow steady motion of a second order thermo-viscous fluid between two parallel plates is examined. The closed form solutions of the velocity and temperature distributions are obtained when thermo-stress coefficient is far less compared to strain thermal conductivity coefficient and coefficient of cross viscosity for the following two cases: (i) when the upper plate is in relative motion and (ii) when the upper plate is thermally insulated. The heat transfer coefficient on the upper plate, The mean Bulk temperature and the transverse force perpendicular to the flow direction are also calculated. It is observed that forces are generated in transverse directions which are special feature of these types of fluids. The effect of various flow parameters on the flow field have been discussed with the help of graphical illustrations.
\end{abstract}

Keywords: Thermo-viscous fluids, Strain thermal conductivity coefficient and Thermo stress Coefficient.

\section{INTRODUCTION}

The non-Newtonian nature of materials has been the subject of extensive study for over one and half centuries. It is only in last seven decades that serious attempts have been made to extend these investigations in the realm of non-linearity. The failure of the linear theories in predicting to a reasonable extent the mechanical behavior of materials such as liquid polymers, fluid plastic, the molten metal's etc subjected to stresses has been the motivating force behind study of the nonlinear theories for material description. A non- linear generalization of the Voigt type materials was proposed by Rivlin [16] and Eringen [4]. Some of the non-liner theories proposed so far (listed in references) have not taken into account the strong dependence of visco-elastic behaviour upon thermal conduction i.e. interaction/interrelation between mechanical and non mechanical (such as thermal, chemical, electromagnetic etc.) effects even though the large amount data of experimental evidence indicate a strong dependence of visco-elastic nature of the fluid upon thermal behavior ( Ferry [5] ).

The development of non-linear theory reflecting the interaction/interrelation between thermal and viscous effects has been preliminarily studied by Koh and Eringen [9] and Coleman and Mizel [3] . A systematic rational approach for such a class of fluids has been developed by Green and Nagdhi [6]. In 1965 Kelly [10] examined some simple shear flows of second order thermo-viscous fluids . Nageswara Rao and Pattabhi Ramacharyulu [14] later studied some steady state problems dealing with certain flows of thermo-viscous fluids. Some more problems K.Anuradha [1] and E.Nagaratnam [12] studied in plane, cylindrical and spherical geometries.

Flows of incompressible homogenous thermo-viscous fluids satisfy the following basic equations.

Equation of Continuity:

$$
v_{i, i}=0
$$

Equation of Momentum:

$$
\rho\left[\frac{\partial v_{i}}{\partial t}+v_{k} v_{i, k}\right]=\rho F_{i}+t_{j i, j}
$$

Equation of Energy:

$$
\rho c \dot{\theta}=t_{i j} d_{i j}-q_{i, i}+\rho \gamma
$$

Where

$F_{i}=i^{t h}$ Component of external force per unit mass

$c=$ Specific heat 
$\gamma=$ Thermal energy source per unit mass

and

$q_{i}=i^{\text {th }}$ Component of heat flux bivector $=\epsilon_{i j k} h_{j k} / 2$

Solving a specific boundary value problem would mean, finding the solution of these equations with appropriate boundary conditions such as the no slip condition (i.e. the velocity of fluid relative to the boundary is zero) and the prescription of the wall temperature. The later condition may be replaced by the prescription of heat flux on the boundary.

\section{MATHEMATICAL FORMULATION}

AND

\section{SOLUTION OF THE PROBLEM}

With reference to the Cartesian coordinates system $\mathrm{O}(\mathrm{x}, \mathrm{y}, \mathrm{z})$ with origin on the lower plate, the $\mathrm{X}$-axis in the direction of flow. The flow is characterized by the velocity field

$[u(y), 0,0]$ and temperature by $\theta(\mathrm{y})$.

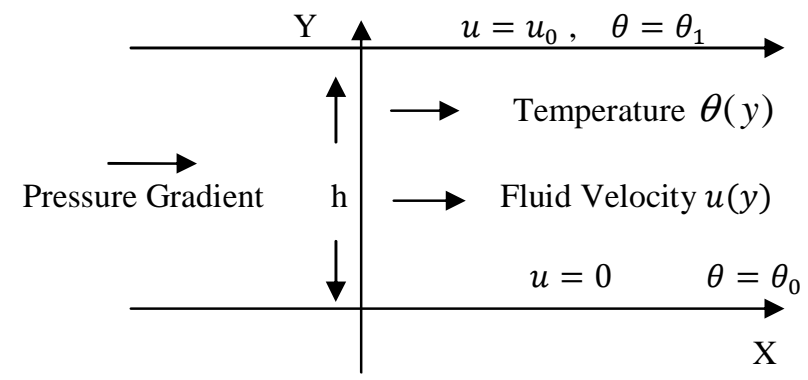

Fig 1: Flow Configuration

In the absence of any external force in the direction of flow, the equations of motion reduces to

$$
\begin{aligned}
& 0=-\frac{\partial p}{\partial x}+\mu \frac{\partial^{2} u}{\partial y^{2}}-\alpha_{6} \frac{\partial \theta}{\partial x} \frac{\partial^{2} \theta}{\partial y^{2}} \\
& \mu_{\mathrm{c}} \frac{\partial}{\partial y}\left(\frac{\partial u}{\partial y}\right)^{2}+\rho \mathrm{F}_{\mathrm{y}=0} \\
& \alpha_{8} \frac{\partial}{\partial y}\left(\frac{\partial \theta}{\partial y} \frac{\partial u}{\partial y}\right)+\rho \mathrm{F}_{\mathrm{z}}=0
\end{aligned}
$$

In the absence of any heat source, the energy equation reduces to

$\rho \mathrm{c}\left(\mathrm{u} \frac{\partial \theta}{\partial x}\right)=\mu\left(\frac{\partial u}{\partial y}\right)^{2}-\alpha_{6} \frac{\partial \theta}{\partial x} \frac{\partial u}{\partial y} \frac{\partial \theta}{\partial y}+\mathrm{k} \frac{\partial^{2} \theta}{\partial y^{2}}+\beta_{3} \frac{\partial \theta}{\partial x} \frac{\partial^{2} u}{\partial y^{2}}$

together with the boundary conditions:

$$
\begin{array}{ll}
u(0)=0, & \theta(0)=\theta_{0} \\
u(h)=u_{0}, & \theta(h)=\theta_{1}
\end{array}
$$

Introducing the following non-dimensional quantities,

$$
y=h Y, u=(\mu / \rho h) U, \quad T=\frac{\theta-\theta_{0}}{\theta_{1}-\theta_{0}}, \frac{\partial \theta}{\partial x}=\frac{\theta_{1}-\theta_{0}}{h} c_{2}
$$

and $\quad-\frac{\partial p}{\partial x}=\frac{\mu^{2}}{\rho h^{3}} c_{1}$

The equation (1) and (4) can be reduced to

$$
0=c_{1}+\frac{d^{2} U}{d Y^{2}}-A_{6} c_{2} \frac{d^{2} T}{d Y^{2}}
$$

and

$$
U c_{2}=A_{1}\left[\left(\frac{\mathrm{d} U}{\mathrm{dY}}\right)^{2}-A_{6} C_{2} \frac{d U}{d Y} \frac{d T}{d Y}\right]+\frac{1}{p_{r}} \frac{d^{2} T}{d Y^{2}}+B_{3} C_{2} \frac{d^{2} U}{d Y^{2}}
$$

With $p_{r}=\frac{c \mu}{k}$ (Prandtle number), $\mathrm{S}=\frac{\rho h v_{0}}{\mu}, \mathrm{B}_{3}=\frac{\beta_{3}}{\rho h^{2} c}$,

$$
\mathrm{A}_{1}=\frac{\mu^{2}}{\rho h^{2} c\left(\theta_{1}-\theta_{0}\right)} \text { and } \mathrm{A}_{6}=\frac{\alpha_{6\left(\theta_{1}-\theta_{0}\right)^{2}}}{\mu^{2}}
$$

where

$C_{1}$ is Non-Dimensional constant pressure gradient and $C_{2}$ is Non-Dimensional constant temperature gradient.

The boundary conditions are $\mathrm{U}(0)=0, \mathrm{U}(1)=U_{0}$

$$
\mathrm{T}(0)=0, \quad \mathrm{~T}(1)=1
$$

\section{CASE-I: WHEN UPPER PLATE IS IN}

\section{RELATIVE MOTION}

Assuming that the thermo - stress coefficient $\alpha_{6}$ is far less when compared to strain thermal conductivity coefficient $\beta_{3}$ and coefficient of cross viscosity $\mu_{\mathrm{c}}$.

The equations (7) and (8) reduces to

$$
0=c_{1}+\frac{d^{2} U}{d Y^{2}}
$$

$U c_{2}=A_{1}\left(\frac{\mathrm{dU}}{\mathrm{dY}}\right)^{2}+\frac{1}{p_{r}} \frac{d^{2} T}{d Y^{2}}+B_{3} C_{2} \frac{d^{2} U}{d Y^{2}}$

The boundary conditions reduces to

$$
\begin{array}{cc}
\mathrm{U}(0)=0, & \mathrm{~T}(0)=0 \\
\mathrm{U}(1)=U_{0}, & \mathrm{~T}(1)=1
\end{array}
$$


i.e. the upper plate is moving with a given velocity and the two plates are maintained at different temperatures.

The equations (9) and (10) together with the boundary conditions (11) and (12) yield the velocity

$$
U(Y)=\frac{C_{1}}{2} Y(1-Y)+U_{0} Y
$$

And the temperature

$$
\begin{aligned}
T(Y)= & Y+A_{1} P_{r}\left[\frac{C_{1}^{2}}{24}\left(2 Y^{2}-2 Y+1\right)+\frac{U_{0}^{2}}{2}-\right. \\
& \text { U0C16 } 2 Y-1 Y 1-Y+C 2 p r \quad \text { C124Y2-Y-1- }
\end{aligned}
$$

$U 061+Y-B 3 C 22 Y 1-Y$

The heat transfer coefficient i.e. Nussult number " $\mathrm{Nu}$ " on the upper plate is

$$
\begin{array}{r}
\mathrm{Nu}=\left(\frac{d T}{d Y}\right)_{Y=1}=-\frac{A_{1} P_{Y}}{24}\left[\left(C_{1}-2 U_{0}\right)^{2}+8 U_{0}^{2}\right]+ \\
\frac{C_{2} P_{r}}{24}\left[C_{1}+8 U_{0}+12 B_{3} C_{2}\right]+1
\end{array}
$$

Which depends on constant pressure gradient $C_{1}$, the relative velocity of upper plate $U_{0}$ and $B_{3}$ the strain thermal conductivity coefficient, constant temperature gradient $C_{2}$ and the prandtle number $p_{r}$.

Also the mean Bulk temperature $=\frac{\int_{0}^{1} U T d Y}{\int_{0}^{1} U d Y}=$

$$
\left[\begin{array}{c}
U_{0}\left\{\begin{array}{c}
1 / 3+A_{1} p_{r}\left(C_{1}^{2} / 480+U_{0}^{2} / 24-U_{0} C_{1} / 360\right) \\
-C_{2} p_{r}\left(C_{1} / 240-U_{0} / 45+B_{3} C_{2} / 24\right)
\end{array}\right\} \\
\left.+C_{1}\left\{\begin{array}{c}
1 / 24+A_{1} p_{r}\left(C_{1}^{2} / 2520+U_{0}^{2} / 120-U_{0} C_{1} / 360\right) \\
-C_{2} p_{r}\left(17 C_{1} / 20160-U_{0} / 240+B_{3} C_{2} / 240\right)
\end{array}\right\}\right) / \\
\left(U_{0}+\frac{C_{1}}{6}\right)
\end{array}\right.
$$

The forces generated in transverse direction are

$$
\begin{gathered}
\rho F_{y}=2 \mu_{c} \frac{\mu^{2}}{\rho^{2} h^{4}}\left[U_{0}+\frac{C_{1}}{2}(2 Y-1)\right] C_{1} \\
\rho F_{z}=\frac{\alpha_{8} \mu\left(\theta_{1}-\theta_{0}\right)}{h^{4} \rho}=
\end{gathered}
$$

$$
\left\{\begin{array}{c}
c_{1}\left\{\begin{array}{c}
1+A_{1} p_{r}\left\{\begin{array}{c}
\frac{C_{1}^{2}}{6}\left(1+6 Y^{2}-8 Y^{3}\right) \\
+\frac{U_{0} C_{1}}{3}\left(\begin{array}{c}
2-9 Y \\
+9 Y^{2}
\end{array}\right)
\end{array}\right\} \\
C_{2} p_{r}\left\{\begin{array}{c}
-\frac{C_{1}}{24}\left(1+6 Y-24 Y^{2}+16 Y^{3}\right) \\
+\frac{U_{0}}{6}\left(1+3 Y-9 Y^{2}\right) \\
-\frac{B_{3} C_{2}}{2}\left(-1+Y+2 Y^{2}\right)
\end{array}\right\} \\
-U_{0} p_{r}\left\{\begin{array}{c}
\frac{C_{1}^{2}}{4}\left(1-4 Y+4 Y^{2}\right)-U_{0}^{2} \\
-3 U_{0} C_{1}(-1+2 Y) \\
A_{1}\{ \\
+C_{2}\left\{\frac{C_{1}}{2}\left(Y-Y^{2}\right)-U_{0} Y\right\} \\
+B_{3} C_{2} Y
\end{array}\right\}
\end{array}\right\}
\end{array}\right.
$$

It is observed that these forces generated in transverse directions depends on the cross viscosity $\mu_{c}$ and on $\alpha_{8}$ the thermo stress viscosity.

\section{CASE-II: WHEN UPPER PLATE IS} INSULATED

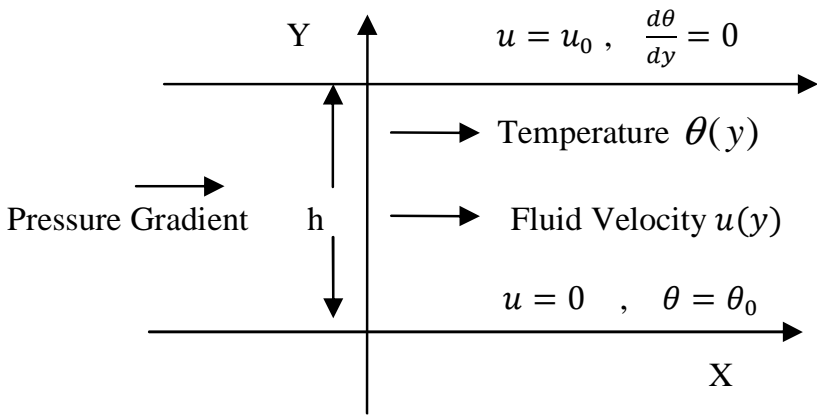

Fig.2: Flow Configuration

The equations of motion and energy reduces to

$$
\begin{aligned}
& 0=c_{1}+\frac{d^{2} U}{d Y^{2}}-A_{6} c_{2} \frac{d^{2} T}{d Y^{2}} \\
& U c_{2}=\mathrm{A}_{1}\left[\left(\frac{\mathrm{dU}}{\mathrm{dY}}\right)^{2}-\mathrm{A}_{6} \mathrm{C}_{2} \frac{d U}{d Y} \frac{d T}{d Y}\right]+\frac{1}{p_{r}} \frac{d^{2} T}{d Y^{2}}+\mathrm{B}_{3} \mathrm{C}_{2} \frac{d^{2} U}{d Y^{2}}
\end{aligned}
$$

Together with the boundary conditions:

$$
\begin{aligned}
& \mathrm{U}(0)=0, \quad \mathrm{~T}(0)=0 \\
& \mathrm{U}(1)=U_{0}, \quad\left(\frac{d T}{d Y}\right)_{Y=1}=0
\end{aligned}
$$


The velocity field is same as in Newtonian case and is given by

$$
U(Y)=\frac{C_{1}}{2} Y(1-Y)+U_{0} Y
$$

And the temperature field is given by

$$
\begin{gathered}
T(Y)=p_{r} C_{2}\left[\begin{array}{c}
\frac{C_{1}}{24}\left(-Y^{3}+2 Y^{2}-2\right)+ \\
\frac{U_{0}}{6}\left(Y^{2}-3\right)
\end{array}\right] Y \\
+A_{1} p_{r}\left[\frac{C_{1}^{2}}{24}\left(2-3 Y+4 Y^{2}-2 Y^{3}\right)-\frac{C_{1} U_{0}}{6}(3-\right. \\
2 Y Y+U 022(2-Y) Y+B 3 C 1 C 2 p r 2(Y-2) Y
\end{gathered}
$$

The forces generated in transverse direction are

$$
\rho F_{y}=2 \mu_{c} \frac{\mu^{2}}{\rho^{2} h^{4}}\left[U_{0}+\frac{C_{1}}{2}(2 Y-1)\right] C_{1}
$$

$$
\begin{aligned}
& \rho F_{z}= \\
& C_{1} \alpha_{8} \mu \theta_{0}\left[\begin{array}{c}
p_{r} C_{2}\left\{\begin{array}{c}
\frac{c_{1}}{12}\left(1-3 Y+6 Y^{2}-4 Y^{3}\right) \\
+\frac{U_{0}}{2}\left(1+Y-3 Y^{2}\right)
\end{array}\right\} \\
+p_{r} \mathrm{~A}_{1}\left[\begin{array}{c}
\frac{C_{1}^{2}}{24}\left(32 Y^{3}-48 Y^{2}+24 Y-5\right) \\
-C_{1} U_{0}\left(-5 Y^{2}+5 Y-1\right)+\frac{U_{0}^{2}}{2}(4 Y-3)
\end{array}\right] \\
+\frac{p_{r} B_{3} C_{1} C_{2}}{2}(3-4 Y) \\
U_{r} C_{2}\left\{\begin{array}{c}
\left.\frac{c_{1}}{12}\left(-Y^{2}+Y\right)+U_{0} Y\right\} \\
-p_{r} \mathrm{~A}_{1}\left[\frac{C_{1}^{2}}{24}\left(4 Y^{2}-4 Y+1\right)+C_{1} U_{0}(1-2 Y)+U_{0}^{2}\right.
\end{array}\right] \\
+p_{r} B_{3} C_{1} C_{2}
\end{array}\right]
\end{aligned}
$$

Which depends on $\alpha_{8}, B_{3}$, pressure gradient, given velocity of upper plate and the constant temperature gradient.

\section{RESULTS AND DISCUSSION}

Numerical estimates of the velocity and temperature fields was carried for different values of $U_{0}=(0,1)$ by taking $C_{1}=1, \mathrm{~A}_{1}=1, p_{r}=1$ and these are illustrated graphically.

When the upper plate is fixed, thermally insulated or not, the velocity is parabolic in general. When the upper plate is in relative motion with a given velocity, the velocity of the fluid is steadily increased so as to attain the velocity of the upper plate, this is observed from the Fig 3.

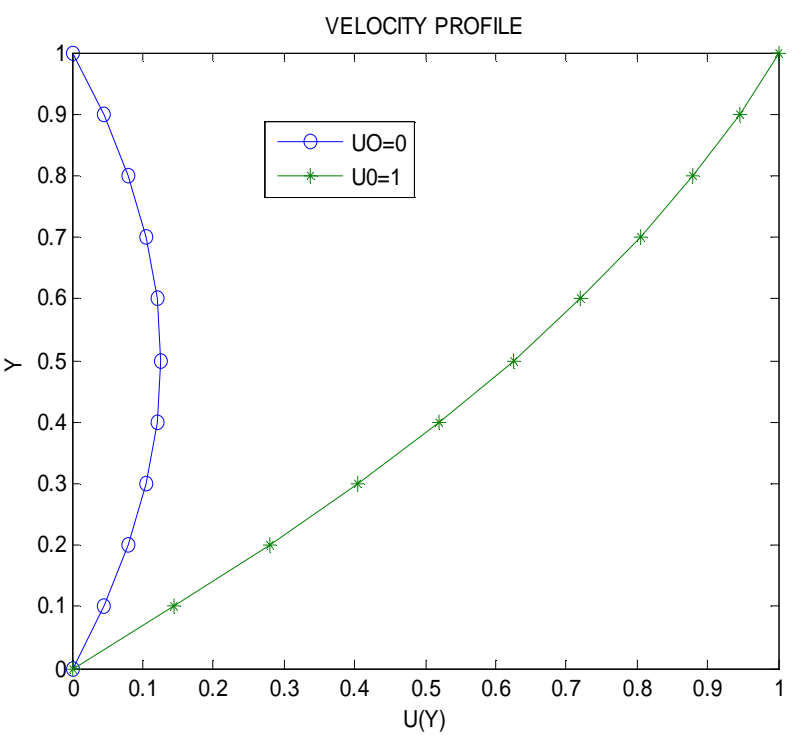

Fig 3: Velocity Profile

The temperature distributions for different values of B3 are illustrated graphically in Fig. 4, 5 and 6

When the upper plate is not thermally insulated temperature increases gradually to attain the temperature of the upper plate and when it is thermally insulated temperature decreases.

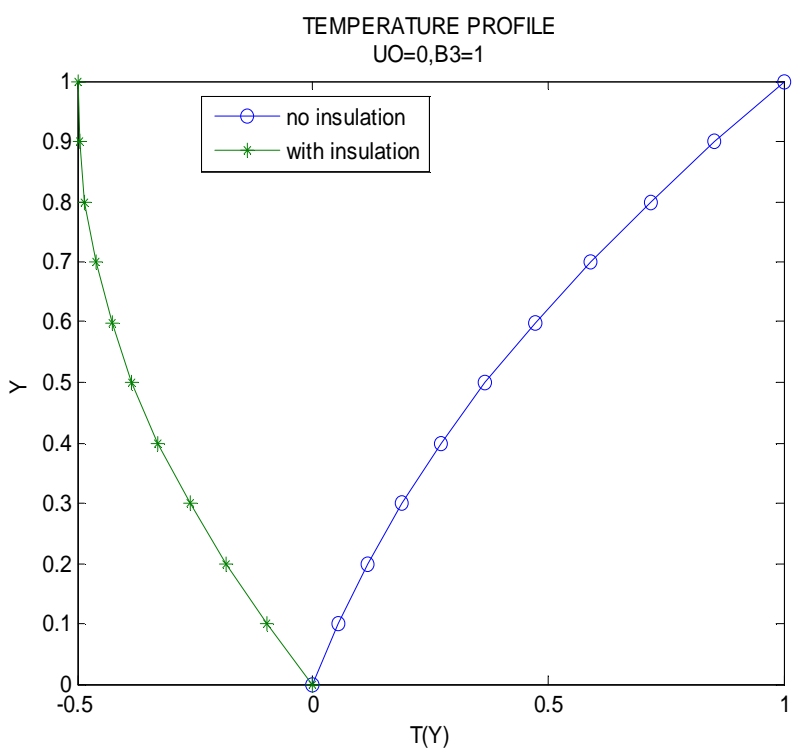

Fig 4: Temperature Profile 


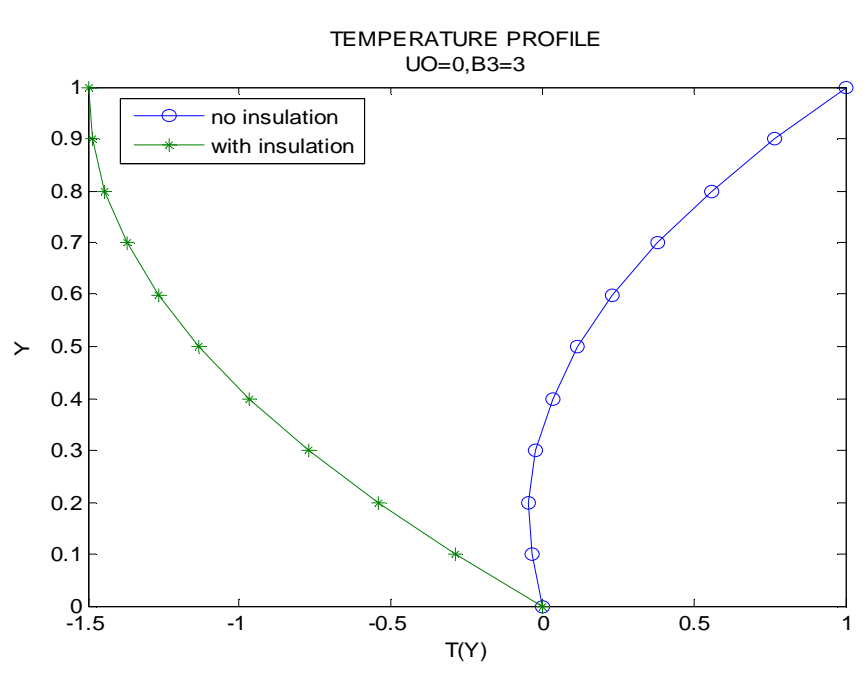

Fig.5: Temperature Profile

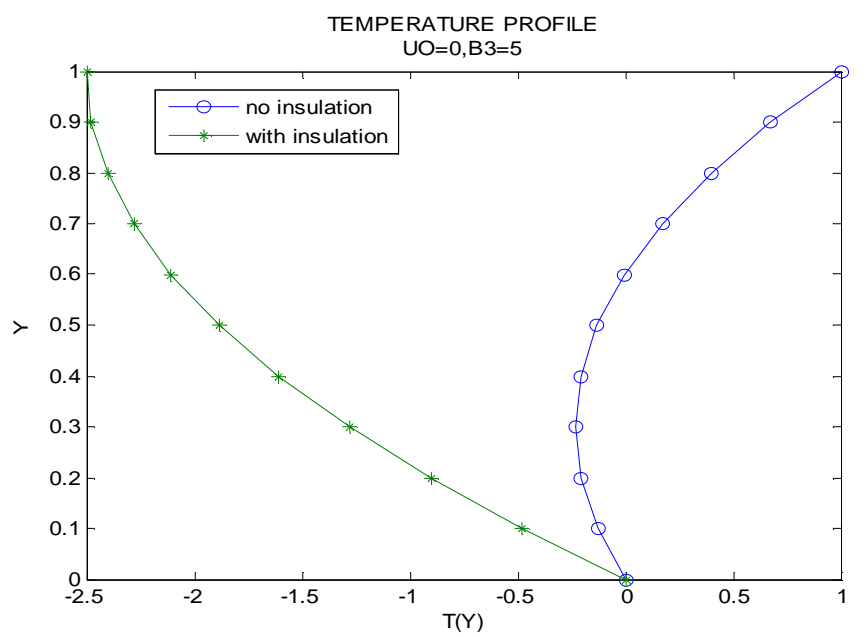

Fig.6: Temperature Profile

When the upper plate is in relative motion, temperature distributions for different values of B3 are illustrated graphically in the figures 7,8 and 9.

When the upper plate is not thermally insulated temperature increases gradually to attain the temperature of the upper plate and when it is thermally insulated temperature decreases.

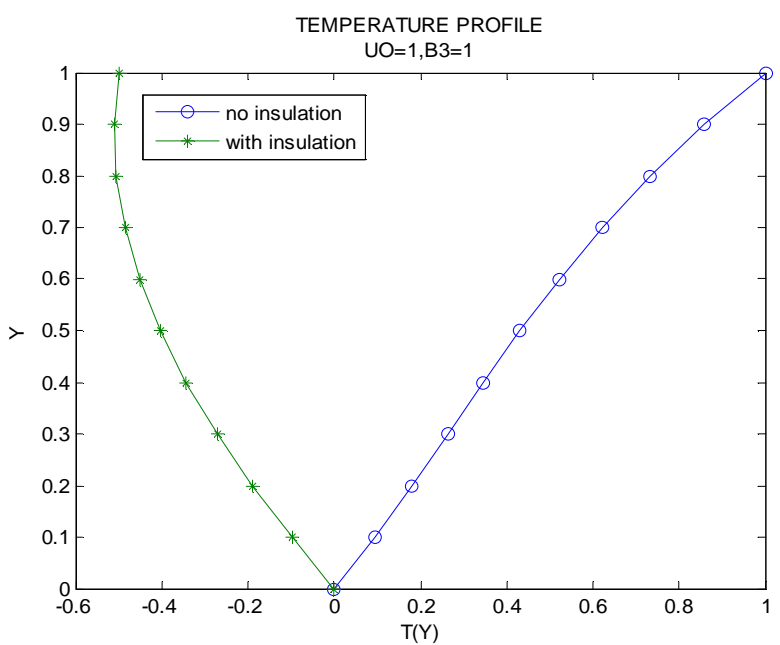

Fig.7: Temperature Profile

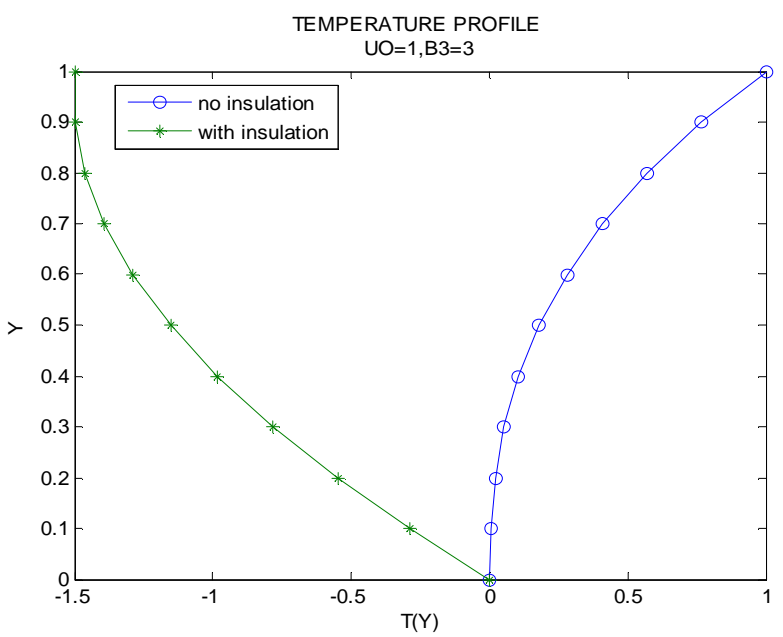

Fig 8: Temperature Profile

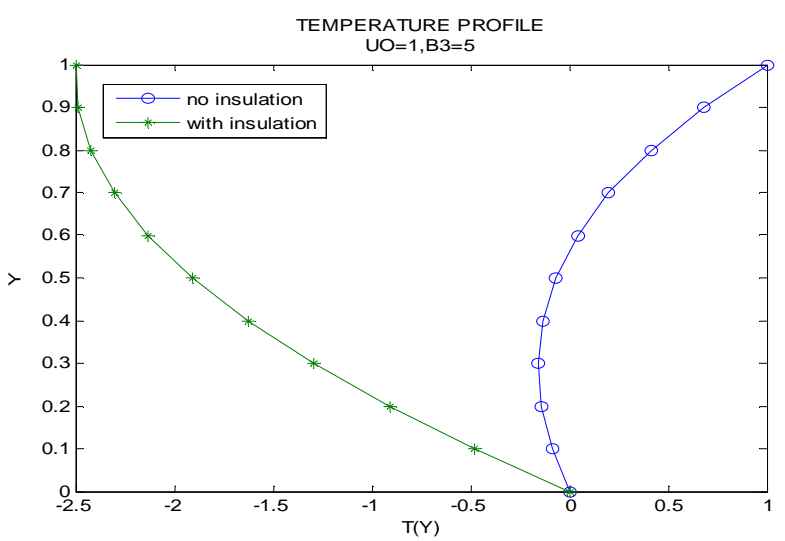

Fig.9: Temperature Profile 


\section{REFERENCES}

[1]. Anuradha , K. , On steady and unsteady flows of thermoviscous Fluids, Ph.D thesis (2006), J.N.T.U. Hyderabad.

[2]. Beaver, G.S and Joseph, D.D. Boundary conditions at a naturally permeable wall, Journal of Fluid Mechanism, Vol.30(1967), pp.197-207.

[3]. Coleman, B.D. and Mizel, V.J. On the existence of caloric equations of state, Journal of Chem.Phys., Vol.40 (1964), pp.1116-1125.

[4]. Eringen ,A.C. Non-linear theory of continuous Media, $M c$ Graw Hill(1962).

[5]. Ferry, J.D. Visco-elastic properties of polymers , Wiley\&Sons(1961), New York.

[6]. Green, A.E. and Naghdi, P.M. A dynamical theory of interacting Continua, Int.J. Engg. Sci. ,Vol.3 (1965), pp. 231241.

[7]. Green, A.E. and Rivlin, R.S. Steady flow of nonNewtonian fluids through tubes, Q.App.Maths. Vol.14(1956), pp.299-308.

[8]. Green, A.E., Rivlin, R.S. and Spencer, A.J.M. The mechanics of non-linear materials with memory-part II, Arch.Rat.Mech.Anal., Vol.4(1959), pp. 82-90.

[9]. Koh, S.L., and Eringen, A.C. on the foundations of nonlinear thermo viscoelasticity, Int.J.Engg.Sci. ,Vol.1(1963), pp.199-229

[10]. Kelly, P. D. Some viscometric flows of incompressible thermo-viscous fluids, Int.J. Engg. Sci. , Vol.2(1965), pp.519537.

[11]. Langlois, W.E., and Rivlin, R.S Slow steady flow of viscoelastic fluids through non-linear tubes. Rendiconti di Mathematica(1963), 22 ,pp.169 [12].

[12].Nagaratnam .E Some steady and unsteady flowsof thermo- viscous fluids, Ph.D thesis (2006), JNTU Hyderabad. [13]. Nageswara rao, p., and Pattabhi Ramacharyulu, N.Ch., Steady flow of thermo-viscous fluids through straight tubes, Journal of IISc. (1979), Vol.61B, NO.6, pp . 89-102.

[14]. Nageswara Rao. P. Some problems in thermo-Viscous fluid Dynamics, Ph.D thesis (1979), K.U. Warangal.

[15]. Pattabhi Ramacharyulu.N.Ch. and Anuradha.K., Steady flow of a thermo- viscous fluid between two parallel plates in relative motion.,Int. J. of Math. sciences, Vol.5(2006).

[16]. Rivlin, R.S., The solution of problems in second order elasticity theory, J.Rat. Mech. Anal. Vol.(3), pp.581-585, (1954) .

[17]. Rivlin, R.S., and Topakaglu, C., A theorem in the theory of finite elastic deformations, J.Rat.Mech.Anal. Vol.(3), pp.581-585, (1954). 